•综述・

\title{
城市化对鸟类筑巢的影响研究综述
}

\author{
蓝方源 1 1\#，马行健 ${ }^{2 \#}$, 逯金瑶 ${ }^{3}$, 李雨果 ${ }^{2}$, 柴汝松 ${ }^{4}$, 李翔 ${ }^{2}$, 罗亦欧 ${ }^{5}$, 张宇泽 ${ }^{2}$, \\ 叶子凌 ${ }^{2}$, 付昌健 ${ }^{2}$, 暴文爽 ${ }^{2}$, 李立 ${ }^{3 *}$, 邢晓莹 ${ }^{2 *}$
}

1. 东北林业大学林学院, 哈尔滨 $150040 ; 2$. 东北林业大学野生动物与自然保护地学院, 哈尔滨 150040; 3. 湖南省生物多样性保护中心, 长 沙 410116; 4. 黑龙江省森林植物园, 哈尔滨 150040; 5. 东北林业大学奥林学院, 哈尔滨 150040

摘要: 繁殖期筑巢是鸟类生活史的重要阶段, 是鸟类繁殖成功的关键保障。全球范围的城市化加速推进, 使城市中筑巢繁殖 的鸟类面临挑战的同时又为其提供了特殊机遇。本文通过搜索现有文献，利用Biblioshiny程序提取并整合关键词，得到城市 鸟类生态研究的热点领域, 分析了城市气候、食物资源、巢址资源、捕食压力、污染和人为干扰对鸟类筑巢的影响，并对今 后城市化对鸟类筑巢影响研究中亟需解决的问题进行了展望。城市化对鸟类筑巢期、巢址选择及巢材选择产生显著影响，与 栖息在村镇生境的鸟类相比，在城市中繁殖的鸟类在筑巢时间、空间和巢材使用上出现变化。在城市中筑巢的鸟类到底是通 过调整行为策略作出积极适应，还是被动接受城市环境中的诸多负面干扰，需要针对研究对象和特定的城市栖息生境进行及 时评估, 而非泛泛之谈。要在研究结论基础上, 深入思考并提出城市化建设过程中有利于鸟类种群和群落保护的具体措施, 高效发挥公民科学作用以解决城市中的鸟类保护实践问题。

关键词: 鸟类; 城市化; 筑巢生态; 营巢期; 巢址选择; 人工巢箱; 巢材

蓝方源, 马行健, 逯金瑶, 李雨果, 柴汝松, 李翔, 罗亦欧, 张宇泽, 叶子凌, 付昌健, 暴文爽, 李立, 邢晓莹 (2021) 城市化对鸟类筑巢的影响 研究综述. 生物多样性, 29, 1539-1553. doi: 10.17520/biods.2021215.

Lan FY, Ma XJ, Lu JY, Li YG, Chai RS, Li X, Luo YO, Zhang YZ, Ye ZL, Fu CJ, Bao WS, Li L, Xing XY (2021) Effects of urbanization on bird nesting: A review. Biodiversity Science, 29, 1539-1553. doi: 10.17520/biods.2021215.

\section{Effects of urbanization on bird nesting: A review}

Fangyuan Lan ${ }^{\oplus 1 \#}$, Xingjian $\mathrm{Ma}^{2 \#}$, Jinyao $\mathrm{Lu}^{3}$, Yuguo $\mathrm{Li}^{2}$, Rusong Chai ${ }^{4}$, Xiang $\mathrm{Li}^{2}$, Yiou Luo ${ }^{5}$, Yuze Zhang ${ }^{2}$, Ziling $\mathrm{Ye}^{2}$, Changjian $\mathrm{Fu}^{2}$, Wenshuang $\mathrm{Bao}^{2}, \mathrm{Li} \mathrm{Li}^{3 *}$, Xiaoying Xing ${ }^{2 *}$

1 College of Forestry, Northeast Forestry University, Harbin 150040

2 College of Wildlife and Protected Area, Northeast Forestry University, Harbin 150040

3 Hunan Biodiversity Conservation Center, Changsha 410116

4 Forest Botanical Garden of Heilongjiang Province, Harbin 150040

5 Aulin College, Northeast Forestry University, Harbin 150040

\section{ABSTRACT}

Background \& Aim: Nesting behavior is important for birds because this stage is critical for breeding success. Since urbanization is expanding worldwide, birds living in cities face huge challenges but the urban environment can also provide living opportunities. This review summarizes bird nesting behaviors in cities, analyzing the influence of factors such as climate, food resources, nest site availability, predation pressure, pollution, and anthropogenic disturbance on bird nesting based on publications in Biblioshiny programme.

Summary: This study demonstrates that urbanization influences bird nesting period, nest site and nest material selection significantly, and that spatio-temporal nesting ranges and nesting materials differ between urban and rural birds. We highlight the need to evaluate whether city birds are well adapted or negatively affected by urbanization, which would require specific analysis of target populations and habitat conditions.

Perspectives: We should propose effective and specific suggestions to protect city birds during urbanization based on

收稿日期: 2021-05-26; 接受日期: 2021-08-10

基金项目：中央高校基本科研业务费专项(2572019BE05)、国家自然科学基金(31501867; 31770454)和东北林业大学大学生创新训练项目 (S202010225153)

\# 共同第一作者 Co-first authors

* 通讯作者 Author for correspondence. E-mail: liliph@163.com; ab71588@163.com 
our research results, instead of simply providing general guidelines. Developing citizen science to solve practical issues in urban animal ecology is a promising direction for the future.

Key words: birds; urbanization; nesting ecology; nest building duration; nest-site selection; artificial nest-box; nest material

随着城市化的快速推进, 越来越多的鸟类栖息 地发生剧变(Shochat et al, 2006)。与自然栖息地相比, 城市景观包含更多建筑和硬化地面, 植被类型与盖 度、捕食者群落都有较大差异(Chace \& Walsh, 2006), 因此城市鸟类栖息地中的食物资源(Crates et al, 2016)、气候 (Davis et al, 2014)、捕食者 (López-Flores et al, 2009)、污染物(Marteinson \& Verreault, 2020)、 干扰源(Sierro et al, 2017)等都会发生显著改变。这 些变化对城市鸟类来说既是生存挑战, 也是拓展新 生态位的机会(Evans et al, 2018)。

筑巢是鸟类繁殖的开始, 对其繁殖成功与否起 关键作用, 需要投入大量时间和能量(Moreno et al, 2008)。城市环境中鸟类的营巢生境与自然栖息地截 然不同, 梳理和分析目前针对城市化对鸟类筑巢影 响的研究, 可更好地理解鸟类为应对城市化而作出 的繁殖策略调整, 同时有助于将已有研究成果应用 于指导城市化管理过程中的鸟类保护, 例如在城市 建设中哪些措施可为鸟类提供更多的营巢机会, 以 提高鸟类种群繁殖力和增加群落多样性。

本文将从以下几个方面总结和探讨城市化对 鸟类筑巢的影响：(1)城市化对鸟类筑巢期的影响 及原因; (2)城市化带来的各种变化如何影响鸟类 的巢址选择及繁殖成效; (3)城市化背景下巢材和 巢外形的变化。最后展望未来城市鸟类筑巢研究的 发展方向。

\section{方法}

于2021年4月通过Web of Science (http://apps. webofknowledge.com)搜集城市化对鸟类筑巢影响 的英文文献, 数据库选择“Web of Science核心合 集”。首先在主题搜索中使用“Bird* OR Avian” AND “Urban* OR City OR Park OR Garden” AND “Nest” 检索; 在标题搜索中使用“Bird* OR Avian” AND “Urban” AND “Breeding* OR Reproduction OR Survival”补充检索。出版年限定在2003-2021年, 文 献类型为“论文”和“综述论文”。搜集的文献通过 Biblioshiny程序进行分析(Aria \& Cuccurullo, 2017;
李昊，2018)，提取每篇文章作者提供的关键词，得 到关键词的频数分布。对关键词进行整合, 去除不 能代表某个具体研究领域的词汇以及主题搜索时 采用的词汇, 合并具有相近词义或是所代表的研 究方向接近的关键词并排序, 最终得到城市鸟类 繁殖生态研究的热点领域, 以筑巢行为作为核心 串联热点领域的主要成果并加以分析。

同时通过中国知网(https://www.cnki.net/)搜集 中文文献, 高级检索中使用主题搜索 “城市化” AND “鸟类” AND “繁殖” OR “筑巢”; 补充搜索时 在高级检索中使用主题搜索“鸟类”, 在结果中使用 标题搜索“巢址选择” OR “人工巢箱” OR “巢捕食” OR “巢材”。发表年度限定在2003-2021年, 文献类 型为“研究论文”和“综述”。保留标题中含有搜索关 键词的文章, 对于标题中不含关键词的文章通过汶 览摘要判断是否保留, 以此作为补充。

\section{城市化对鸟类筑巢繁殖影响的主要研究方｜}

剔除无关文献后共收集到英文文献1,451篇 中 文文献62篇，提取并整合后得到关键词31个，按内 容分为 10 个研究方向。涵盖方向包括城市鸟类的生 境变化、群落和种群特征、繁殖生态、筑巢行为、 捕食、干扰等(表1)。

对 10 个研究方向中的关键词频数进行统计, 发 现当前对城市化与鸟类筑巢生态关系的研究更多 聚焦于鸟类的栖息生境、繁殖、种群和群落多样性 以及行为。栖息地环境是动物适应性和生物多样性 保护研究中最重要的内容, 而且城市生境中的廊 道、斑块格局的多样化等生态学特征也极富研究价 值(Wu, 2004), 例如城市中小尺度的生境可能更加 复杂(Pennington \& Blair, 2011)。目前, 以城市鸟类 为对象的研究更关注市区和郊区的生境差异与鸟 类繁殖成效差异间可能存在的关联与影响 (Chamberlain et al, 2009; Seress et al, 2018), 而在种 群与群落多样性层次则更偏重分析城郊不同景观 中鸟类种群波动、群落结构与生态系统之间的相互 
表1 城市鸟类繁殖生态研究关键词频数排序

Table 1 Frequency sorting of urban bird breeding ecology research keywords

\begin{tabular}{|c|c|c|c|c|}
\hline $\begin{array}{l}\text { 研究方向 } \\
\text { Research area }\end{array}$ & $\begin{array}{l}\text { 包含的关键词* } \\
\text { Key words* }\end{array}$ & $\begin{array}{l}\text { 关键词频数 } \\
\text { Frequency }\end{array}$ & $\begin{array}{l}\text { 占比 } \\
\%\end{array}$ & $\begin{array}{l}\text { 排名 } \\
\text { Ranking }\end{array}$ \\
\hline 生境 Habitat & $\begin{array}{l}\text { 景观、破碎化、生境选择、植被结构 } \\
\text { Landscape, fragmentation, habitat selection, and vegetation structure }\end{array}$ & 156 & 20.2 & 1 \\
\hline 繁殖 Breeding & $\begin{array}{l}\text { 繁殖生态学、繁殖成功率、窝卵数、育维数 } \\
\text { Breeding biology, reproductive success, clutch size, and brood size }\end{array}$ & 115 & 14.9 & 2 \\
\hline 群落 Community & $\begin{array}{l}\text { 群落结构、物种丰富度、多样性 } \\
\text { Community structure, species richness, and diversity }\end{array}$ & 102 & 13.2 & 3 \\
\hline 捕食 Predation & $\begin{array}{l}\text { 捕食风险、被捕食、巢捕食 } \\
\text { Predation risk, prey, and nest predation }\end{array}$ & 96 & 12.4 & 4 \\
\hline 种群 Population & $\begin{array}{l}\text { 种群动态、种群趋势、丰度、死亡率 } \\
\text { Population dynamics, population trends, abundance, and mortality }\end{array}$ & 85 & 10.9 & 5 \\
\hline 行为 Behavior & $\begin{array}{l}\text { 扩散、竞争、入侵、迁徙、受食行为、模式 } \\
\text { Dispersal, competition, invasions, migratory, foraging behavior, and } \\
\text { patterns }\end{array}$ & 75 & 9.7 & 6 \\
\hline 巢址选择 Nest-site selection & 巢址选择、人工巢 Nest-site selection and artificial nests & 48 & 6.2 & 7 \\
\hline 土地利用 Land uses & 土地利用、住宅开发 Land uses and residential development & 40 & 5.2 & 8 \\
\hline 食物 Food & 食物 Food & 32 & 4.1 & 9 \\
\hline 人为干扰 Disturbance & 人为干扰、噪声、光照 Human disturbance, noise, and light & 25 & 3.2 & 10 \\
\hline
\end{tabular}

*已合并具有相近词义或是所代表的研究方向接近的关键词

*We have merged keywords that have similar meanings or represent similar research directions

作用(Vigallon \& Marzluff, 2005)。此外，城市景观中 野生鸟类的食物源、巢址与人为干扰等因素与自然 环境相比都有所不同(Evans et al, 2018), 因此相关 研究领域包括了城市中生存的鸟类在受食、巢址选 择、反捕食以及应对人为干扰等方面的适应性 (Scales et al, 2011; Narango et al, 2017; Møller \& Díaz, 2018; Jokimäki et al, 2020)。

\section{3 城市化对筑巢期的影响}

筑巢期是反映鸟类适应当地环境的主要生活 史特征之一, 对城市鸟类筑巢期的研究不仅有助于 完善鸟类繁殖生物学, 也可为解构鸟类的生态适 应提供重要线索(Deviche \& Davies, 2015; 蒋政权 等, 2017)。下面将从温度、食物等因素出发, 总结 分析城市化对鸟类筑巢期开始时间和长度的影响。

\section{1 城市化对筑巢开始时间的影响}

开始筑巢是鸟类启动繁殖的标志之一, 选择何 时开始筑巢是实现繁殖成功的关键 (Both et al, 2006)。研究发现城市鸟类存在繁殖期提前的现象, 但此类研究通常是以产首枚卵的日期来衡量繁殖 期开始时间(Chamberlain et al, 2009), 未能很好地 描述城市化对鸟类繁殖期的影响是对整个繁殖过 程还是仅对某个繁殖阶段。因此, 本节将尝试区分 能够影响包括筑巢期在内整个繁殖过程的以及可
能仅对筑巢期有明确影响的城市化因素, 并探讨两 者对筑巢期开始时间的影响。前者指能够让鸟类提 前达到繁殖所需生理状态的城市化因素, 后者指可 能仅为筑巢行为提供便利的因素。

城市化影响鸟类筑巢时间的因素是多样的, 能 够直接影响鸟类生理状态的因素包括温度、光照和 食物资源等(Chamberlain et al, 2009; Deviche \& Davies, 2015)。城市热岛效应(urban heat island)带来 的城市温度升高是否会直接影响筑巢期, 还缺乏确 業的证据(Deviche \& Davies, 2015)。但已有研究发 现人工光源可能会刺激鸟类性腺发育进而增加其 繁殖行为表达的概率(Dawson et al, 2001), 致使鸟 类提前开始出现鸣唱、筑巢等繁殖行为(Deviche \& Davies, 2015)。城市中稳定且长期存在的人工食源 也会使鸟类更早地达到繁殖所需生理状态(Crates et al, 2016)。这些城市化因素并不单一作用于某个繁 殖阶段, 而是通过影响鸟类的生理状态使繁殖期提 前开始，带动了各个繁殖阶段的开始时间。

城市化还可能通过影响植物和无脊椎动物的 物候间接改变鸟类开始筑巢的时间。城市中较高的 温度使得鸟类可更早地获取无脊椎动物食物 (Forrest, 2016; Chick et al, 2019), 有证据表明城市 中某些鸟类繁殖时间与鳞翅目幼虫数量高峰相对 应(Hajdasz et al, 2019)。Hajdasz等(2019)发现城市中 
繁殖的北美白眉山雀(Poecile gambeli)开始筑巢时 间大约提前了一周, 这可能是因为热岛效应使城市 中植物物候提前(Parece \& Campbell, 2018), 导致山 雀繁殖期主要食源鳞翅目幼虫数量高峰提前出现 (Marie et al, 2016)。但或许因为不同研究地的城市 植被结构与覆盖度不同, 也有相反的研究结果 (Seress et al, 2018)。例如有些城市绿化面积过少, 造 成昆虫数量较少(Fenoglio et al, 2020), 该情况下鸟 类的繁殖行为或许不会密切配合鳞翅目幼虫的爆 发期(Wawrzyniak et al, 2015), 即使食物数量高峰提 前出现也无法促使城市鸟类提前繁殖(Hajdasz et al, 2019)。因此研究过程中应留意研究地岛类种群与其 食源的特定关系, 繁殖行为与食源的关系紧密与否 可能会导致不同的研究结果。且该类研究主要思路 是“城市增温-植物提前萌发一植食性昆虫提前出现 数量高峰-食虫鸟类提前筑巢” (Seress et al, 2018), 对植物类食源占比较大的鸟类则研究较少。值得一 提的是, 一般认为对同一鸟种而言, 分布在较高纬 度的个体筑巢时间延后(朱䂀等, 2000)。但在对白头 嘟(Pycnonotus sinensis)的研究中却发现高纬度的城 市个体(四川、浙江)筑巢时间早于低纬度非城市个 体(广西)的情况(刘小华和龙国珍, 1986; 陈伟等, 2006; 张琴, $2014^{\circledR}$ ), 暗示热岛效应可能会减小纬度 对鸟类筑巢时间的影响。城市化与纬度梯度这两种 因素对鸟类筑巢或繁殖的协同作用应在未来研究 中加以关注。

城市鸟类提前筑巢还可能与适宜巢址资源出 现更早有关, 这种改变主要作用于筑巢期而非整个 繁殖阶段。在罗骏等(2008)的研究中, 市区繁殖的乌 冻(Turdus mandarinus)主要选择在常年郁闭度良好 的常绿乔木香樟(Cinnamomum camphora)上筑巢; 郊区繁殖的乌鸫主要以落叶乔木白杨 (Populus tomentosa) 作为巢树。由于巢树的郁闭度与鸟类巢 址的安全性关系密切(施丽敏等, 2012), 郊区的乌鸫 需等待巢树长出新叶达到一定的郁闭度, 所以筑巢 开始时间会相对延后(罗骏等, 2008)。此外, 即使巢 树类型相同, 市区中的巢树也可能因热岛效应而提 前发芽、展叶(赖小红等, 2019), 使适宜巢址资源出 现得更早。虽然该研究未与郊区相应的常绿树种中

(1) 张琴 (2014) 白头鹎生活史特征对城市化的响应. 硕士学位论 文, 浙江师范大学, 浙江金华.
的乌冻进行对比, 不能排除城乡之间其他差异对筑 巢开始时间的影响, 但为细化鸟类筑巢期研究提供 了一个新思路。需要注意的是, 鸟类会根据树冠发 育程度选择筑巢树种(Ludvig et al, 1995), 例如金翅 雀(Chloris sinica)在繁殖初期偏爱利用常绿针叶树 作为巢树, 可能因为此时落叶阔叶树尚未或刚进入 展叶期, 而常绿树能提供更好的隐蔽性; 繁殖中后 期随着落叶阔叶树郁闭度增加, 选择在树上筑巢的 金翅雀也逐渐增加(马龙等, 2020)。Kosiński (2001) 对欧金翅雀(Chloris chloris)的研究也发现了这一现 象。这种行为可能会在一定程度上减小筑巢树种对 鸟类筑巢开始时间的影响, 使得城市鸟类提前筑巢 现象不甚明显。

综上, 城市鸟类提前筑巢可能的原因可概括为: (1)城市中的光照、温度和食物资源对鸟类生理有直 接或间接的影响, 从而诱发鸟类繁殖提前。(2)城市 与非城市环境植被类型之间的差异以及热岛效应, 造成两者的植物物候期不同, 导致可供鸟类利用的 巢址资源出现时间不同。未来研究应进一步区分不 同食性的鸟类对城市化的响应, 并尝试量化城市化 与纬度梯度这两种因素对鸟类筑巢影响的贡献程 度。但由于城市化过程中常伴随着大片的绿地改造, 且北方城市不断引入南方植物种类 (Qian et al, 2016), 这种跨气候区的城市绿地同质化(Wheeler et al, 2017)或许会使得城市化与纬度梯度的影响更难 区分。此外, 筑巢期提前这一现象是否会导致物候 匹配错误(Hajdasz et al, 2019)从而降低城市鸟类的 繁殖成效, 仍值得深入研究。

\section{2 城市化对筑巢期长度的影响}

筑巢期长度是衡量鸟类繁殖投入的重要指标 之一(Álvarez \& Barba, 2008), 反映了鸟类的繁殖策 略(Berg et al, 2006)。从资源供给的角度看, 城市化 或许会使鸟类筑巢期缩短。首先, 城市中充足且稳 定的食源(Crates et al, 2016)使得鸟类在筑巢期不需 要花费太多时间和精力受食, 可以将更多时间用于 筑巢而缩短筑巢期(Mainwaring \& Hartley, 2008)。同 时, 城市中丰富的人造材料为衔材提供了便利(李 音等, 2017), 也可能导致筑巢期缩短(Mainwaring \& Hartley, 2013)。

但从压力胁迫的角度看, 结果又有所不同。鸟 类在城市中某些地区面临着很高的捕食威胁(Loss \& Marra, 2017), 而巢捕食会对鸟类的繁殖策略产 
生重要影响。在巢捕食威胁较高的生境, 鸟类会减 少当次繁殖投入, 例如减少窝卵数(Hua et al, 2014), 以留存更多时间和能量用于再次筑巢 (Martin, 1995)。此外, 亲鸟还会减少回巢次数以减小巢捕食 威胁(Mutzel et al, 2013)。但相关研究集中于产卵、 孵卵和育维期, 所以这种减少繁殖投入的策略是从 筑巢开始并持续整个繁殖季, 还是仅在产卵之后开 始尚不清楚。如果是前者, 则鸟类可能会在筑巢时 减少时间投入从而导致筑巢期缩短, 也可能因减少 回巢次数而延缓筑巢进度致使筑巢期延长。

此外, 巢体积和巢型也会影响筑巢期长短( 蒋 政权等, 2017), Mainwaring和Hartley (2013)的研究 发现, 乌冻筑巢期长度与巢大小正相关。但关于城 市化对巢体积与质量影响的研究较少(Hanmer et al, 2017; Lambrechts et al, 2017), 目前暂无定论。

总之, 关于城市化对鸟类筑巢期长度的影响, 还需要大量基础研究作为证据。对于城市鸟类筑巢 期的研究应更明确地区分城市化在不同进程、不同 繁殖阶段的影响, 是持续整个繁殖过程还是仅对某 个繁殖阶段有影响, 这种更为细化的研究有助于更 清楚地了解城市对鸟类繁殖的具体影响以及鸟类 的应对策略。

\section{4 城市中鸟类甾址的改变}

鸟类巢址选择是与环境协同进化而形成的繁 殖对策, 巢址质量对鸟类繁殖成功至关重要 (Millones \& Frere, 2017)。城市景观结构与鸟类自然 栖息地相差很大, 主要表现在城市建筑物数量和密 度远远超过自然栖息地、植被种类趋于单一(MacGregor-Fors \& Schondube, 2011)、植被呈现聚集型 分布(如公园、人工绿化林等) (谢世林等, 2016)、捕 食者群落改变(Jokimäki et al, 2020)、存在大量流浪 猫(Sims et al, 2008)及人类干扰增多(Scales et al, 2011)等。本节将从城市建筑与人工巢箱、城市植被、 捕食者压力及人为干扰等方面总结城市化对鸟类 巢址选择的影响。

\section{1 城市建筑}

鸟类利用城市建筑是对城市化的适应性表现 (Schnack, 1991)。城市建筑可分为建筑物和构筑物, 两者区别主要在于前者可供人居住，一般指楼房; 后者包括电线杆、桥梁、道路、人造洞穴等 $(G B / T$ 50504-2009)。城市中的建筑物为鸟类提供了新的巢
址资源, 如房屋外墙的空调孔洞和窗沿墙缝, 这些 孔道和缝隙为诸如普通雨燕(Apus apus)、紫翅椋鸟 (Sturnus vulgaris)、麻雀(Passer montanus)和家燕 (Hirundo rustica)等鸟类提供了筑巢场所(Chace \& Walsh, 2006; Mainwaring, 2015; 李翔等, 2019); 水 体附近的建筑物屋顶为很多水鸟提供了筑巢平台, 如蚛璚(Haematopus ostralegus) (Munro, 1984)和鸥 类(Soldatini et al, 2008)等。能够适应并积极利用此 类巢址的鸟类通常会有更高的繁殖成效(王龙祥等, 2020), 进而逐渐扩大种群规模和分布范围(De Laet \& Summers-Smith, 2007)。例如Schnack (1991)发现 城区的欧乌斩(Turdus merula)比同域分布的欧歌鸫 (T. philomelos)能更多地利用建筑巢址, 由此在城区 繁殖密度更高。并且人为干扰降低了捕食风险 $(\mathrm{St}$ Clair et al, 2010), 这使得在人类居住地建筑物上筑 巢的鸟类往往会有较弱的反捕食行为(Møller \& Díaz, 2018), 从而有更多精力投入繁殖。但随着城 市化进程加快, 现代化建筑逐渐取代旧建筑, 而现 代高层建筑大多外表平滑整齐, 缺少孔洞缝隙和支 撑点, 缺乏可供鸟类使用的巢基附着物和筑巢场所 (张淑萍等, 2006)。此外, 对于部分利用旧巢的鸟类 而言, 城市化的加剧不仅会减少潜在巢址, 老旧城 区改造和商业区重新装修还可能会破坏旧巢址(植 飞等, 2018)。

此外, 建筑物楼道封闭情况可能会直接影响巢 址选择。例如, 封闭的阳台或窗户阻碍家燕出入, 且居民对家燕筑巢的容忍度也是影响家燕巢址选 择的重要因素(植飞等, 2018; 李翔等, 2019)。这提 示我们在研究城市鸟类时要更重视建筑物使用情 况以及当地居民的态度, 这些微小的差异或许会导 致生活在不同地区的同一鸟种采取不同的筑巢策 略。

城市中大量的构筑物也为鸟类提供了重要筑 巢场所(Mainwaring, 2015; Duckworth et al, 2017), 但这也产生了许多生态陷阱 (ecological traps) (Schlaepfer et al, 2002; Ben-Aharon et al, 2020)。椋鸟 科、鸦科以及部分水鸟和猛禽会选择电线杆和高架 塔作为巢址(Dixon, 2013; 李阳林等, 2017), 这会增 加鸟类触电死亡的风险(Jenkins et al, 2010; Kaluga et al, 2011)。巢址资源紧张可能导致巢较为简单的 鸟类(如鸽形目鸟类)在人造物突起上筑巢, 易遭到 人为破坏或移除, 导致鸟类繁殖成效降低 
(Schlaepfer et al, 2002; 张琴等, 2013)。路面和桥梁 同样为很多鸟类提供了筑巢场所, 其中道路由于边 缘效应, 具有较复杂的植被结构吸引鸟类在此繁殖 (高鹏等, 2012), 而强烈的人为干扰会使雉鸟死亡率 升高(Ben-Aharon et al, 2020)。但也有学者认为是由 于当地巢址有限导致鸟类不得不选择较为嘈杂的 路面或桥梁筑巢(Reynolds et al, 2019)。鸟类对巢址 的选择是主动还是被动, 在一定程度上反映了鸟类 对城市化的适应程度。

对于城市建筑与鸟类巢址关系的研究, 应该更 多地关注不同研究地建筑制式以及巢址的空间特 征, 包括但不限于: (1)巢址空间的大小、形状; (2) 有无合适的支撑物或筑巢平台; (3) 出入口是否通 畅。在以往的研究中, 学者们往往更关注巢址的分 布和微气候对鸟类繁殖的影响, 而较少考虑筑巢空 间在周围环境中的地位。例如适宜巢址在研究地的 丰富程度、是否容易被鸟类或捕食者发现, 以及人 类对该空间的态度和控制程度等。

\section{2 人工巢箱}

城市中常悬挂人工巢箱和筑巢平台招引鸟类, 在鸟类研究与保护中使用越来越广泛(Olah et al, 2014), 包括雀形目(Duckworth et al, 2017)、鹳形目 (Tryjanowski et al, 2009)和鸮形目(Smith et al, 2005) 等, 且有证据表明人工巢箱可能比自然巢址更受欢 迎(Rodríguez et al, 2011), 可能是因其可减少亲鸟 对营巢的投入而提高繁殖成功率(Teglhøj, 2018)。

但人工巢箱在使用中会产生一系列新问题。首 先, 人工巢箱可能会吸引鸟类到不适宜的繁殖地点 而成为生态陷阱(Klein et al, 2007), 但也有长期监 测发现人工巢箱确实促进了目标种群的增长 (Sutherland et al, 2014)。其次, 捕食者对人工巢箱内 鸟卵或雉鸟的捕食与自然巢相比还缺乏经验 (Mitrus, 2003), 而捕食者在积累经验后是否会增加 捕食却尚不清楚。例如布设在某地区的巢箱大小或 高度通常相似(Stagoll, 2012), 这种外形和空间位置 上的同质化可能导致巢箱更容易被捕食者发现, 增 加巢捕食风险。

从巢箱种类来看, 传统的胶合板或木制巢箱不 能有效地模仿树洞环境条件(Maziarz, 2017), 且巢 箱对于缓冲极端气候表现较差, 巢内微气候条件较 之天然巢更为极端(Griffiths et al, 2018), 可能成为 潜在的生态陷阱。在对蓝胸佛法僧 (Coracias garrulus)的研究中发现尽管人工巢箱内小气候条件 更极端, 但人工巢箱与天然巢的繁殖参数相似。蓝 胸佛法僧会选择缓冲能力最好的巢箱从而避免潜 在的生态陷阱(Schwartz et al, 2020), 这一发现有利 于优化人工巢箱的布置。某些对小气候变化不敏感 的物种可能无法避开那些潜在生态陷阱的巢址, 而 对那些能辨别低质量巢箱的鸟类而言, 人工巢箱确 实可以缓解巢址资源紧张的状况(Schwartz et al, 2020)。但对于其他鸟种来说, 人工巢箱的负面影响 很难在短期内被发现(Valera et al, 2019), 能否促进 种群增长还需长期监测。

相较于胶合板巢箱, 直接在树干上制造人工树 洞可以提供更稳定的巢内环境(Griffiths et al, 2018), 且人工树洞在结构上与自然树洞更相似(Rueegger, 2017), 更容易被目标动物接受和使用(Griffiths et al, 2018), 同时还不易引起捕食者注意。这种方法在针 对某些野生动物的保护计划中被证实是有效的, 例 如红顶豚木鸟(Leuconotopicus borealis) (Cox \& McCor-mick, 2016)。尽管具备更好的使用效果和更 低成本(Rueegger, 2017), 但人工树洞尚未被广泛应 用(Griffiths et al, 2018), 这或许与城市中缺少高大 树木有关(Liu et al, 2019)。

人工巢箱对鸟类的保护有积极作用, 在用于城 市绿地时(Goldingay et al, 2018), 应考虑上述提及 的诸多弊端。并且, 无论是出于生物防治目的还是 鸟类生态学研究目的, 都不应该滥用巢箱。巢箱所 带来的潜在生态陷阱需要警惕, 但此方面的研究多 针对个体而非种群, 或是仅涉及巢捕食率、繁殖成 效等, 缺少对整个种群增长状况长期监测与评估, 所以巢箱的负面效果是否会影响到目标种群的保 护仍不得而知。同时, 并不是所有巢箱的使用者都 是目标招引鸟类(Shuttleworth, 2001), 鸟类是否会 使用被捕食者造访过的巢箱, 也值得深入探讨。

\section{3 城市植被}

不管是在城市还是在自然环境中, 安全性(如 巢高、隐蔽度等)都是影响鸟类巢址选择的关键因素, 反映在巢树选择上则是偏好具有一定高度且郁闭 度良好的乔木或灌木(孟晓静等, 2014)。而暿鸟 (Pica pica)和红嘴蓝鹊(Urocissa erythrorhyncha)等体型较 大鸟类会对巢树胸径有要求, 偏好高大的乔木(高 远等, 2014; 于学伟等, 2014)。但在城市化过程中很 多本土植物被移除, 引进较单一的外来植物作为绿 
化植被(Narango et al, 2017), 改变了城市绿地植被 群落的年龄结构, 使得老龄且高大的树木减少(徐 超, $2012^{\circledR}$ ), 有证据表明城市中缺少高大树木是部 分鸟类转而在建筑上筑巢的重要原因 (Wiebe, 2011)。而成熟空心树木减少意味着城市环境无法提 供充足且足够大的树洞(Lindenmayer \& Laurance, 2017), 这对一些体型较大的次级洞巢鸟(secondary cavity nesters)是不利的, 如红角鸮(Otus scops)和领 角鸮(O. bakkamoena)等小型鸮类(李玲玉等, 2015), 而目前人工巢箱还无法很好地替代这类天然树洞 (Le Roux et al, 2016)。

植被群落的改变会影响部分岛类的繁殖成效, 研究发现主红雀(Cardinalis cardinalis)在外来植被 中的巢捕食率增加, 繁殖成功率降低, 原因可能是 外来植物与本土植物的差异使其更容易被捕食者 发现(Rodewald et al, 2010)。外来植物的增加还可能 导致当地植食性昆虫减少(Narango et al, 2017), 从 而无法为幼鸟提供充足的基础营养(Ortega et al, 2006), 这或许是许多城市鸟类繁殖出飞率低于非 城市鸟类的原因(Chamberlain et al, 2009)。但也有研 究表明在外来植物中筑巢的鸟类与在本土植物中 筑巢的鸟类具有相似的繁殖成效 (Schlossberg \& King, 2010; Meyer et al, 2015), 原因可能是不同生 态系统中外来植物对本地野生动物的影响不尽相 同(Stinson \& Pejchar, 2018)。

城市化带来的城市绿地岛屿化和碎片化使鸟 类栖息地发生显著变化, 并产生诸多不利影响 (Maina \& Jackson, 2003), 例如增加了巢捕食(孙吉 吉等, 2011)和种间巢寄生(如棕头牛丽 Molothrus ater) (Burhans \& Thompson, 2006)的风险。此外, 由 于城市内硬化地面的增加, 众多鸟类个体被挤压在 有限的繁殖位点(人工绿化带、城市公园等), 形成高 密度巢现象(谢世林等, 2016; Han et al, 2019)。有学 者认为这种集群分布是鸟类适应城市化的对策之 一(田军东等, 2009), 诸如喜鹊、夜鹭(Nycticorax nycticorax) 等鸟类在城市中的筑巢密度增大(杨月伟 和慈海金金, 2005; 陈侠斌等, 2006), 加剧了鸟类对巢 址的竞争, 同时也促进形成集体繁殖领域以应对城 市中的生存压力。

(1) 徐超 (2012) 南京市城市绿地结构动态变化的研究. 硕士学位 论文, 南京林业大学, 南京.
城市植被一直是城市鸟类栖息地研究的重要 一环, 已有大量研究探讨了城市植被组成、结构、 空间分布与鸟类筑巢的关系。在此基础上, 应从生 态系统网络的视角出发优化城市绿地设计(赵伊琳 等, 2021), 缓解鸟类巢址和高质量食物资源缺乏的 压力。同时, 在对外来植物的研究中应该区分生物 入侵与绿化引种, 并明确其处于何种入侵阶段。

\section{4 捕食者压力和人为干扰}

城市中繁殖的鸟类要面对不同于自然栖息地 的筑巢环境、捕食者群落(Jokimäki et al, 2020)和人 为干扰(Scales et al, 2011), 这些变化可能会对鸟类 巢址选择产生显著影响(Xu et al, 2020)。城市鸟类面 临的捕食压力是否增加仍存在较大争议 (López-Flores et al, 2009)。一般认为城市中的天敌 较少, 故巢捕食率较低(Sorace, 2002)。但也有基于 人工巢实验的研究表明市中心和居民区的捕食者 数量比乡村或林区更多(Jokimäki et al, 2005), 主要 原因是有大量流浪猫(Felis catus)分布(蒋志刚和郭 晓辉, 2007), 而猫是城市鸟类的主要捕食者之一 (Sims et al, 2007; Li et al, 2021)。

人为干扰被认为是影响城市鸟类分布的另一 主要因素(陈水华等, 2000, 2002)。繁殖期鸟类对人 为干扰更为敏感(王彦平等, 2003), 可直接影响其巢 址选择(张琴等, 2013)。但是, 充分适应城市生活的 鸟类会选择在人类活动频繁的区域筑巢以避开巢 捕食者(Møller \& Díaz, 2018; 张雷等, 2020), 有证 据表明适应人类活动而在人为干扰更强区域筑巢 的鸟类比在远离人类区域繁殖的同类繁殖成效更 高(Møller \& Díaz, 2018; 王龙祥等, 2020)。有些鸟种 会升高巢位以降低被地面捕食者发现的概率 (Rendell \& Robertson, 1989), 规避捕食者和人为干 扰(Xu et al, 2020), 已有大量研究发现巢高与城市 化正相关(吕艳等, 2008)。例如, 红尾伯劳(Lanius cristatus) (徐纯柱和郭自荣, 2011)、白㚘噪鸤 (Pterorhinus sannio) (严勇等, 2016)、白头䅉(兰思思 等, 2013)、喜鹊(吕艳等, 2008)等。

为应对捕食者压力与人为干扰, 城市鸟类主要 采取调整巢址与巢高的策略。由于城市间景观与植 被群落结构差异较大, 捕食者压力的高低受研究地 具体状况以及研究尺度影响。整体上看城市内巢捕 食压力更小, 且人类活动减弱了鸟类反捕食行为, 但在流浪猫大量分布区域(居民区、公园)会有较高 
的巢捕食风险。对此城市鸟类会以升高巢位来适应 人为干扰和改变了的捕食者群落。城市绿地面积、 垂直结构、空间布局对城市鸟类躲避捕食者、降低 人为干扰, 最终成功繁殖尤为重要。如果城市景观 中的斑块-廊道一踏脚石空间布局合理，鸟类也能在 景观中适宜生境比例低的情况下维持生存(Melles et al, 2003)。未来的研究可以尝试切换角度, 从捕食 者的视角研究城市化对鸟类筑巢的影响, 或许能够 揭示更真实的巢捕食影响因素, 以及城市鸟类针对 捕食者作出的巢址选择策略调整。此外, 由于人为 干扰的特殊性, 城市生态研究过程中应该更重视基 于公民科学和人文背景的调查。

\section{城市化对巢材和㧺结构的影响}

自然栖息地中鸟类使用的巢材基本来自本土 植物和野生动物毛发等, 附近的优势植物往往是鸟 巢中所占比例较大的巢材(丁志锋等, 2020)。而城 市 鸟类面对一系列全新的巢材来源, 多来自绿化植 被、人造材料、废弃物、人类毛发等(Radhamany et al, 2016; Reynolds et al, 2016)。本节将总结城市化导 致巢材改变的原因假说以及巢结构(体积与重量)的 改变及其可能原因。

(1)可得性假说(availability hypothesis): 该假说 假设鸟类会优先选择最容易获得的材料, 就近取材 以保留更多能量用于繁殖后代(Mennerat et al, 2009)。有学者认为城市中鸟类巢材改变是由于城市 内本土植物的减少和人造材料的增加(Wang et al, 2009)。例如: 黑脸琵鹗(Platalea minor)在巢周围的 自然材料增加后减少使用人造巢材; 大山雀(Parus major)在巢材中增加使用主要绿化树种松树的松针, 而松针并不是自然条件下常用巢材 (Lambrechts et al, 2017); 白㚘噪鵰在巢材中增加了人类生活材料, 且巢材组成变化与城市绿化植被的改变有关(李桂 垣, 1982; 严勇等, 2016)。

(2)年龄增长假说(age hypothesis): 该假说认为 城市中定居的鸟类会随年龄增长而逐渐增加使用 人造材料(Jagiello et al, 2018), 其中原因尚不清楚, 但可能是基于老龄岛的经验(Reynolds et al, 2019)。

(3)适应假说 (adaptive hypothesis): 该假说认为 鸟类巢材及结构的改变是由自身的适应性驱动的, 以应对城市环境中的压力。例如, 部分鸟类会在巢 材中使用烟头(Suárez-Rodríguez \& Garcia, 2017)和
艾草(Yang et al, 2020) 以有效减少寄生虫数量 (Hanmer et al, 2017), 并提高繁殖成效 (SuárezRodríguez \& Garcia, 2014)。该行为被认为是一种自 我治疗(Clayton \& Wolfe, 1993)。另外, 热岛效应也 可能是导致鸟类减少使用保温巢材 (毛发和羽毛等) 的原因(Reynolds et al, 2019)。

(4)新位置假说(new location hypothesis): 该假 说认为巢材改变是对新巢址环境的适应(Reynolds et al, 2016)。例如在窗沿上筑巢的乌冻和在烟图或 房顶筑巢的喜鹊(Wang et al, 2008, 2015), 由于有稳 定底座而减少了巢材的使用, 此外城市内巢箱的使 用也可能会改变鸟类对巢材的需求(Wesołowski, 2011)。

可得性假说和年龄增长假说都假定鸟类使用 人造材料是因为其与自然巢材相似(Townsend \& Barker 2014; Biddle et al, 2018), 可在一定程度上替 代自然巢材。年龄增长假说暗示了人造材料比自然 材料更适宜, 可能是因为人造材料易于获得(李音 等, 2017); 而适应假说认为城市提供了具有某些特 性(如抗菌性)的材料, 在适应性的驱动下鸟类使用 了这些材料; 新位置假说则认为筑巢环境的改变产 生了新的需求, 以致于城市鸟类改变巢材。使用人 造材料也可能产生不利后果, 例如塑料绳、渔网、 塑料碎片等物品可能会导致误食或缠绕而危害鸟 类繁殖与健康(Townsend \& Barker, 2014; Seacor et al, 2014; Provencher et al, 2017), 尤其是海鸟 (Jagiello et al, 2018)。此外, 烟头中的毒性物质也会 对鸟类产生影响(Suárez-Rodríguez et al, 2017)。

巢结构的改变也是城市化对鸟类筑巢影响的 一个重要方面, 但目前研究结果各不相同(Hanmer et al, 2017; Lambrechts et al, 2017)。关于城市鸟巢的 大小和重量, 一些学者发现蓝山雀 (Cyanistes caeruleus)或者大山雀的城市鸟巢体积和重量与自 然栖息地中无显著差异 (Glądalski et al, 2016; Lambrechts et al, 2017), 但也有学者发现城镇中蓝 山雀的巢比自然环境中更轻(Hanmer et al, 2017)。这 可能与前文所提到的城市热岛效应有关, 因为山雀 类鸟巢结构设计主要取决于当地的温度(Deeming et al, 2012)。此外, 鸟巢主要由附着层、外层、结构 层和内层 4 个部分组成, 而相关研究常不加以区分 (Wang et al, 2009), 或是仅关注内层(Reynolds et al, 2019), 这可能是由于鸟巢的内层使用的人工材料 
更多(Reynolds et al, 2019)。

虽然城市鸟类巢材和巢结构这一领域已有大 量研究, 但随着城市化的持续推进, 可供鸟类选择 的巢材也在不断变化, 因此, 对城市鸟类巢材和巢 结构应保持关注。此外, 在以往的研究中常忽略发 展速度对鸟巢的影响。在一些发展较快的地区，鸟 类可能来不及对城市化作出响应, 尤其是在对比中 外鸟类筑巢生态学研究时应格外注意这一点。

\section{城市化对鸟类繁殖成效的影响}

目前已有较多研究对比城市和自然环境(或郊 区) 中筑巢鸟类的繁殖成效 (Chamberlain et al, 2009)。㱠如城市鸟类窝卵数通常更少(Mennechez \& Clergeau, 2006), 这可能是城市化带来的压力迫使 鸟类改变了繁殖策略(Chamberlain et al, 2009; Hua et al, 2014), 而压力一般来自捕食者(Martin, 1995) 和人为干扰(Scales et al, 2011)或是低质量的食物、 巢址资源(Kristan \& Boarman, 2007; Wiebe, 2011)。 同时, 有许多研究发现在城市中繁殖的鸟类倠鸟数 量和出飞率更低, 例如乌冻、紫翅椋鸟、蓝山雀等 (Chamberlain et al, 2009), 其原因或许与城市中强 烈的人为干扰(Ben-Aharon et al, 2020)以及大量外 来植物(Rodewald et al, 2010)有关。此外, 原本在城 市中有较高繁殖成效的鸟类随着城市化进程逐渐 加深，其繁殖成效可能降低(Peach et al, 2008)。

值得注意的是, 城市化带来的改变是多方面的, 在对比不同研究的结果时需要先明确研究地对鸟 类繁殖成效起主要控制作用的特定因素是否一致, 但这通常因为部分文献缺乏相关描述而无法系统 地分析与讨论。

\section{7 展望}

城市和乡村都有种类和数量较多的鸟类栖息, 是了解城市化对野生动物影响的极佳素材。种内和 种间的城乡对比, 有助于揭示鸟类对城市化的适应 性策略。目前城市化对鸟类影响的相关研究虽较为 丰富, 涵盖行为学、生态学、系统进化等多方面, 但 对鸟类筑巢行为生态学的相关研究仍然较缺乏, 对 城市鸟类的繁殖生态学研究的了解仍不够全面, 未 来可着重从以下几个方面深入开展探讨。

(1)目前更多的研究关注区域尺度上, 即不同 纬度对鸟类繁殖期的影响, 而忽略了局地气候环境
的改变也会对鸟类繁殖时间产生影响, 以及城市化 对繁殖的影响是否涵盖整个繁殖过程, 或是对不同 繁殖阶段有不同影响。

(2)了解城市鸟类是否有合适且充足的巢址对 于城市鸟类保护尤为重要。部分鸟类对巢址的特殊 需求和潜在的生态陷阱, 需要野生动物保护和管理 部门在制定相关决策时重点考虑。城市化带来的巢 址资源紧张可通过使用人工巢箱和人工树洞缓解, 但可能会使鸟类身陷更大的巢捕食风险或其他潜 在生态陷阱, 因此该方法在实践中的有效性依然有 待检验, 且应该以种群数量的多年波动情况作为评 判标准。

(3)鸟类巢材对城市化的响应会随着城市化进 程而改变, 因此, 针对巢材与巢外形和结构的持续 研究仍然是必要的, 将有助于更好地了解鸟类在城 市中筑巢所作出的适应性调整。同时对孵化期和育 雏期进行详细记录, 可揭示人造材料与巢结构改变 对繁殖产生的更深远影响。

(4)不同程度城市化地区的鸟类筑巢行为也需 要更为细化的研究, 以探索鸟类在不同城市化梯度 的背景下适应策略的差异。同时, 研究地的发展历 史也应纳入考量范围, 发展速度不同的城市给当地 野生鸟类施加的压力存在差异, 在发展速度较快的 城市中生活的鸟类可能缺乏足够的时间作出响应, 这可能是某些研究结果(捕食者压力、巢结构、繁殖 成效等)存在差异乃至相反的原因。此外, 随着城市 化进程的推进与更多鸟类选择在城市定居繁殖, 人 与野生鸟类接触也越发频繁, 人鸟共患病的预防或 成未来城市公共卫生安全的一大挑战。而如何在 “人鸟冲突”中兼顾人类利益和鸟类保护, 仍是未来 城市规划需要考虑的问题。

\section{ORCID}

蓝方源 (1D) https://orcid.org/0000-0002-4748-8934 邢晓莹 (D) https://orcid.org/0000-0001-7739-9046

\section{参考文献}

Álvarez E, Barba E (2008) Nest quality in relation to adult bird condition and its impact on reproduction in great tits Parus major. Acta Ornithologica, 43, 3-9.

Aria M, Cuccurullo C (2017) bibliometrix: An R-tool for comprehensive science mapping analysis. Journal of Informetrics, 11, 959-975.

Ben-Aharon N, Kapota D, Saltz D (2020) Roads and road-posts 
as an ecological trap for cavity nesting desert birds. Frontiers in Conservation Science, 1, 614899.

Berg ML, Beintema NH, Welbergen JA, Komdeur J (2006) The functional significance of multiple nest-building in the Australian reed warbler Acrocephalus australis. Ibis, 148, 395-404.

Biddle LE, Broughton RE, Goodman AM, Deeming DC (2018) Composition of bird nests is a species-specific characteristic. Avian Biology Research, 11, 132-153.

Both C, Bouwhuis S, Lessells CM, Visser ME (2006) Climate change and population declines in a long-distance migratory bird. Nature, 441, 81-83.

Burhans DE, Thompson FR III (2006) Songbird abundance and parasitism differ between urban and rural shrublands. Ecological Applications, 16, 394-405.

Chace JF, Walsh JJ (2006) Urban effects on native avifauna: A review. Landscape and Urban Planning, 74, 46-69.

Chamberlain DE, Cannon AR, Toms MP, Leech DI, Hatchwell BJ, Gaston KJ (2009) Avian productivity in urban landscapes: A review and meta-analysis. Ibis, 151, 1-18.

Chen SH, Ding P, Fan ZY, Zheng GM (2002) Selectivity of birds to urban woodlots. Zoological Research, 23, 31-38. (in Chinese with English abstract) [陈水华, 丁平, 范忠勇, 郑 光美 (2002) 城市鸟类对斑块状园林栖息地的选择性. 动物学研究, 23, 31-38.]

Chen SH, Ding P, Zheng GM, Zhuge Y (2000) Impacts of urbanization on the wetland waterbird communities in Hangzhou. Zoological Research, 21, 279-285. (in Chinese with English abstract) [陈水华, 丁平, 郑光美, 诸葛阳 (2000) 城市化对杭州市湿地水鸟群落的影响研究. 动物 学研究, 21, 279-285.]

Chen W, Guo ZM, Hu JC, Yu ZW (2006) Breeding habit, growth and development of Pycnonotus sinensis in Nanchong, China. Chinese Journal of Zoology, 41, 107-111. (in Chinese with English abstract) [陈伟, 郭宗明, 胡锦惪, 余志伟 (2006) 四川南充市白头殅的繁殖习性及雉鸟的 生长发育. 动物学杂志, 41, 107-111.]

Chen XB, He J, Zhang W (2006) Ecological factors affecting choice of Pica pica sericea nest location in universities in Beijing. Sichuan Journal of Zoology, 25, 855-857, 861. (in Chinese with English abstract) [陈侠斌, 何静, 张薇 (2006) 北京高校喜鹊巢址选择的主要生态因素. 四川动 物, 25, 855-857, 861.]

Chick LD, Strickler SA, Perez A, Martin RA, Diamond SE (2019) Urban heat islands advance the timing of reproduction in a social insect. Journal of Thermal Biology, 80, 119-125.

Clayton DH, Wolfe ND (1993) The adaptive significance of self-medication. Trends in Ecology \& Evolution, 8, 60-63.

Cox JA, McCormick JK (2016) New insights from an attempt to reintroduce red-cockaded woodpeckers in northern Florida. Journal of Field Ornithology, 87, 360-370.

Crates RA, Firth JA, Farine DR, Garroway CJ, Kidd LR, Aplin LM, Radersma R, Milligan ND, Voelkl B, Culina A,
Verhelst BL, Hinde CA, Sheldon BC (2016) Individual variation in winter supplementary food consumption and its consequences for reproduction in wild birds. Journal of Avian Biology, 47, 678-689.

Davis AY, Malas N, Minor ES (2014) Substitutable habitats? The biophysical and anthropogenic drivers of an exotic bird's distribution. Biological Invasions, 16, 415-427.

Dawson A, King VM, Bentley GE, Ball GF (2001) Photoperiodic control of seasonality in birds. Journal of Biological Rhythms, 16, 365-380.

De Laet J, Summers-Smith JD (2007) The status of the urban house sparrow Passer domesticus in north-western Europe: A review. Journal of Ornithology, 148, 275-278.

Deeming DC, Mainwaring MC, Hartley IR, Reynolds SJ (2012) Local temperature and not latitude determines the design of blue tit and great tit nests. Avian Biology Research, 5, 203-208.

Deviche P, Davies S (2015) Reproductive phenology of urban birds: Environmental cues and mechanisms. In: Avian Urban Ecology (eds Gil D, Brumm H), pp. 98-115. Oxford University Press, New York.

Ding ZF, Liang JC, Feng YJ, Liu JC, Hu HJ (2020) Functional and phylogenetic diversity of birds in urban habitat patches in Macao, China. Chinese Journal of Ecology, 39, 1238-1247. (in Chinese with English abstract) [丁志锋, 梁 健超, 冯永军, 刘金成, 胡慧建 (2020) 澳门城市栖息地 斑块中鸟类群落功能和谱系多样性. 生态学杂志, 39, 1238-1247.]

Dixon A, Purev-Ochir G, Galtbalt B, Batbayar N (2013) The use of power lines by breeding raptors and corvids in Mongolia: Nest-site characteristics and management using artificial nests. Journal of Raptor Research, 47, 282-291.

Duckworth RA, Hallinger KK, Hall N, Potticary AL (2017) Switch to a novel breeding resource influences coexistence of two Passerine birds. Frontiers in Ecology and Evolution, $5,72$.

Evans BS, Reitsma R, Hurlbert AH, Marra PP (2018) Environmental filtering of avian communities along a ruralto-urban gradient in Greater Washington, D.C., USA. Ecosphere, 9, e02402.

Fenoglio MS, Rossetti MR, Videla M (2020) Negative effects of urbanization on terrestrial arthropod communities: A meta-analysis. Global Ecology and Biogeography, 29, 1412-1429.

Forrest JR (2016) Complex responses of insect phenology to climate change. Current Opinion in Insect Science, 17, 49-54.

Gao P, Jin YH, Wang JT, Song ZF, Cao YP, Zhou HC (2012) Influence of road utilization on plant and soil in road effect zone in Changbai Mountain Natural Reserve. Ecological Science, 31, 473-480. (in Chinese with English abstract) [高 鹏, 金永焕, 王景田, 宋铸福, 曹艳萍, 周海城 (2012) 长白山自然保护区道路使用对路域植物和土壤的影响. 生态科学, 31, 473-480.] 
Gao Y, Ci HX, Lei XS (2014) Nest-site selection of Pica pica and Cyanopica cyana in protection forest of freeways. Ecological Science, 33, 904-908. (in Chinese with English abstract) [高远, 慈海金金, 雷晓水 (2014) 高速公路防护林 内喜鹊和灰喜鹊的巢址选择. 生态科学, 33, 904-908.]

Glądalski M, Bańbura M, Kaliński A, Markowski M, Skwarska J, Wawrzyniak J, Zieliński P, Cyżewska I, Bańbura J (2016) Effects of nest characteristics on reproductive performance in blue tits Cyanistes caeruleus and great tits Parus major. Avian Biology Research, 9, 37-43.

Goldingay RL, Thomas KJ, Shanty D (2018) Outcomes of decades long installation of nest boxes for arboreal mammals in southern Australia. Ecological Management \& Restoration, 19, 204-211.

Griffiths S, Lentini P, Semmens K, Watson S, Lumsden L, Robert K (2018) Chainsaw-carved cavities better mimic the thermal properties of natural tree hollows than nest boxes and log hollows. Forests, 9, 235.

Hajdasz AC, Otter KA, Baldwin LK, Reudink MW (2019) Caterpillar phenology predicts differences in timing of mountain chickadee breeding in urban and rural habitats. Urban Ecosystems, 22, 1113-1122.

Han YQ, Bai JP, Zhang Z, Wu T, Chen P, Sun GL, Miao LW, Xu ZF, Yu LJ, Zhu CY, Zhao DQ, Ge G, Ruan LZ (2019) Nest site selection for five common birds and their coexistence in an urban habitat. Science of the Total Environment, 690, 748-759.

Hanmer HJ, Thomas RL, Beswick GJF, Collins BP, Fellowes MDE (2017) Use of anthropogenic material affects bird nest arthropod community structure: Influence of urbanisation, and consequences for ectoparasites and fledging success. Journal of Ornithology, 158, 1045-1059.

Hua FY, Sieving KE, Fletcher RJ, Wright CA (2014) Increased perception of predation risk to adults and offspring alters avian reproductive strategy and performance. Behavioral Ecology, 25, 509-519.

Jagiello ZA, Dylewski Ł, Winiarska D, Zolnierowicz KM, Tobolka M (2018) Factors determining the occurrence of anthropogenic materials in nests of the white stork Ciconia ciconia. Environmental Science and Pollution Research, 25, 14726-14733.

Jenkins AR, Smallie JJ, Diamond M (2010) Avian collisions with power lines: A global review of causes and mitigation with a South African perspective. Bird Conservation International, 20, 263-278.

Jiang ZG, Guo XH (2007) Sampling survey of feral cats in urban Beijing. Chinese Journal of Wildlife, 28(1), 3-6. (in Chinese with English abstract) [蒋志刚, 郭晓辉 (2007) 北 京市区流浪猫密度的初步抽样调查. 野生动物, 28(1), 3-6.]

Jiang ZQ, Li FS, Ran JH, Liu W, Zhao CH, Zhang B, Li H (2017) Nest building duration and its contributing factors for black-necked cranes (Grus nigricollis) at Ruoergai, Sichuan, China. Acta Ecologica Sinica, 37, 1027-1034. (in Chinese with English abstract) [蒋政权, 李凤山, 手江洪, 刘伟, 赵 晨皓, 张波, 李华 (2017) 若尔盖湿地保护区黑颈鹤巢期 及影响因子. 生态学报, 37, 1027-1034.]

Jokimäki J, Kaisanlahti-Jokimäki ML, Sorace A, FernándezJuricic E, Rodriguez-Prieto I, Jimenez MD (2005) Evaluation of the "safe nesting zone" hypothesis across an urban gradient: A multi-scale study. Ecography, 28, 59-70.

Jokimäki J, Suhonen J, Benedetti Y, Diaz M, KaisanlahtiJokimäki ML, Morelli F, Pérez-Contreras T, Rubio E, Sprau P, Tryjanowski P, Ibánez-Álamo JD (2020) Land-sharing vs. land-sparing urban development modulate predator-prey interactions in Europe. Ecological Applications, 30, e02049.

Kaługa I, Sparks TH, Tryjanowski P (2011) Reducing death by electrocution of the white stork Ciconia ciconia. Conservation Letters, 4, 483-487.

Klein Á, Nagy T, Csörgő T, Mátics R (2007) Exterior nestboxes may negatively affect barn owl Tyto alba survival: An ecological trap. Bird Conservation International, 17, 273-281.

Kosiński Z (2001) Effects of urbanization on nest site selection and nesting success of the greenfinch Carduelis chloris in Krotoszyn, Poland. Ornis Fennica, 78, 175-183.

Kristan WB, Boarman WI (2007) Effects of anthropogenic developments on common raven nesting biology in the West Mojave Desert. Ecological Applications, 17, 1703-1713.

Lai XH, Li MY, Liu C, Zhong YH, Lin L, Wang HY (2019) The phenological responses of plants to the heat island effect in the main urban area of Chongqing. Acta Ecologica Sinica, 39, 7025-7034. (in Chinese with English abstract) [赖小红, 李名扬, 刘聪, 钟雨航, 林立, 王海洋 (2019) 植物物候对重庆主城区热岛效应的响应. 生态学报, 39, 7025-7034.]

Lambrechts MM, Charmantier A, Demeyrier V, Lucas A, Perret S, Abouladzé M, Bonnet M, Canonne C, Faucon V, Grosset S, Prado G, Lidon F, Noell T, Pagano P, Perret V, Pouplard S, Spitaliéry R, Bernard C, Perret P, Blondel J, Grégoire A (2017) Nest design in a changing world: Great tit Parus major nests from a Mediterranean city environment as a case study. Urban Ecosystems, 20, 1181-1190.

Lan SS, Zhang Q, Huang Q, Chen SH (2013) Breeding ecology of Chinese Bulbul in the urban environment of Hangzhou, China. Zoological Research, 34, 182-189. (in Chinese with English abstract) [兰思思, 张琴, 黄秦, 陈水华 (2013) 杭 州城市环境中白头唡的繁殖生态. 动物学研究, 34, 182-189.]

Le Roux DS, Ikin K, Lindenmayer DB, Bistricer G, Manning AD, Gibbons P (2016) Enriching small trees with artificial nest boxes cannot mimic the value of large trees for hollow-nesting birds. Restoration Ecology, 24, 252-258.

Li GY (1982) Preliminary studies on the breeding behaviour of the white-browed laughing thrush. Zoological Research, 3, 245-251. (in Chinese with English abstract) [李桂垣 (1982) 白顿噪鹛繁殖习性的初步观察. 动物学研究, 3, 245-251.] Li H (2018) Scientific measurement and visualization analysis 
on the research of international scientific research evaluation based on biblioshiny. Journal of Library and Information Science, 3, 36-44. (in Chinese with English abstract) [李昊 (2018) 国际科研评价研究的科学计量及可视化分析一一 基于biblioshiny程序. 图书情报导刊, 3, 36-44.]

Li LY, Wang HT, Li ZK, Yao JY, Wang LM, Jiang YL (2015) Effects of changing nest site resources on raptor community structure. Journal of Northeast Normal University (Natural Science Edition), 47, 102-107. (in Chinese with English abstract) [李玲玉, 王海涛, 李振奎, 姚纪元, 王利民, 姜 云垒 (2015) 巢址资源变化对猛鿖群落结构的影响. 东 北师大学报(自然科学版), 47, 102-107.]

Li X, Tan XP, Zhao L, Chen RH, Fu CJ, Xiang CX, Yuan LC, Ye ZY, Xing XY (2019) Breeding biology of the barn swallow Hirundo rustica mandschurica in Harbin City, China. Chinese Journal of Zoology, 54, 793-799. (in Chinese with English abstract) [李翔, 谭霄鹏, 赵否, 陈睿 涵, 付昌健, 向晨旭, 袁立成, 叶紫芸, 邢晓莹 (2019) 哈尔滨家燕mandschurica亚种繁殖生态特征. 动物学杂 志, 54, 793-799.]

Li Y, Zhang L, Zhang CY, Shu XT, Li DL, Yin JX, Wan DM (2017) Effects of nest construction cost and nest materials on reproduction of Parus major. Chinese Journal of Ecology, 36, 1667-1671. (in Chinese with English abstract) [李音, 张雷, 张聪芗, 舒雪桐, 李东来, 殷江霞, 万冬梅 (2017) 筑巢投入与巢材组成对大山雀繁殖产出的影响. 生态学杂志, 36, 1667-1671.]

Li YH, Wan Y, Shen H, Loss SR, Marra PP, Li ZQ (2021) Estimates of wildlife killed by free-ranging cats in China. Biological Conservation, 253, 108929.

Li YL, Xu N, Zhang Y, Zhi YJ, Shao MQ (2017) The analysis on ecological habits of common birds in electric transmission lines from Jiangxi Province. Journal of Jiangxi Normal University (Natural Science Edition), 41, 510-515, 520. (in Chinese with English abstract) [李阳林, 徐宁, 张 宇，植毅进，邵明勤 (2017) 江西省输电线路常见鸟类习 性分析. 江西师范大学学报(自然科学版), 41, 510-515, 520.]

Lindenmayer DB, Laurance WF (2017) The ecology, distribution, conservation and management of large old trees. Biological Reviews of the Cambridge Philosophical Society, 92, 1434-1458.

Liu JJ, Lindenmayer DB, Yang WJ, Ren Y, Campbell MJ, Wu CP, Luo YQ, Zhong L, Yu MJ (2019) Diversity and density patterns of large old trees in China. Science of the Total Environment, 655, 255-262.

Liu XH, Long GZ (1986) Preliminary observation of breeding habits of Chinese bulbul. Chinese Journal of Zoology, 21, 12-15. (in Chinese) [刘小华, 龙国珍 (1986) 白头軠繁殖 习性的初步观察. 动物学杂志, 21, 12-15.]

López-Flores V, Macgregor-Fors I, Schondube JE (2009) Artificial nest predation along a Neotropical urban gradient. Landscape and Urban Planning, 92, 90-95.

Loss SR, Marra PP (2017) Population impacts of free-ranging domestic cats on mainland vertebrates. Frontiers in Ecology and the Environment, 15, 502-509.

Lü Y, Zhang YX, Sai DJ, Zhou YP (2008) Nest-selection and urban environment adaption of magpie. Sichuan Journal of Zoology, 27, 892-893. (in Chinese with English abstract) [吕艳, 张月侠, 赛道建, 周玉朋 (2008) 喜鹊巢位选择对 城市环境的适应. 四川动物, 27, 892-893.]

Ludvig E, Vanicsek L, Torok J, Csorgo T (1995) The effect of nest-height on the seasonal pattern of breeding success in blackbirds Turdus merula. Ardea, 83, 411-418.

Luo J, Li YH, Hu J (2008) Nest site choice of Turdus merula sowerbyi in farmland area of Nanchong, Sichuan. Sichuan Journal of Zoology, 27, 575-578. (in Chinese with English abstract) [罗骏, 李艳红, 胡杰 (2008) 四川南充农田区乌 冻的巢址选择. 四川动物, 27, 575-578.]

Ma L, Bai JP, Lu R, Zhang PQ, Chang H, Li LJ, Shi HQ (2020) Nest-site selection of grey-capped greenfinch in east Gansu, China. Chinese Journal of Ecology, 39, 3303-3310. (in Chinese with English abstract) [马龙, 柏军鹏, 路瑞, 张佩 琪, 常慧, 李莲吉, 史红全 (2020) 陇东地区金翅雀的巢 址选择. 生态学杂志, 39, 3303-3310.]

Macgregor-Fors I, Schondube JE (2011) Gray vs. green urbanization: Relative importance of urban features for urban bird communities. Basic and Applied Ecology, 12, 372-381.

Maina GG, Jackson WM (2003) Effects of fragmentation on artificial nest predation in a tropical forest in Kenya. Biological Conservation, 111, 161-169.

Mainwaring MC (2015) The use of man-made structures as nesting sites by birds: A review of the costs and benefits. Journal for Nature Conservation, 25, 17-22.

Mainwaring MC, Hartley IR (2008) Seasonal adjustments in nest cup lining in blue tits Cyanistes caeruleus. Ardea, 96, 278-282.

Mainwaring MC, Hartley IR (2013) The energetic costs of nest building in birds. Avian Biology Research, 6, 12-17.

Marie V, Frank A, Alexandr A, Jerzy B, Emilio B, Clotilde B, Jacques B, Zihad B, Jean-Charles B, Jordi C, Francesco C, Anne C, Motti C, Mariusz C, Camillo C, Dorota C, Virginie D, Blandine D, Claire D, Anna D, Marcel E, Tapio E, Bruno F, Peter FN, Jukka FT, Eduardo GDR, Aya G, Anne GE, Andrew GG, Arnaud, GG (2016) Interspecific variation in the relationship between clutch size, laying date and intensity of urbanization in four species of hole-nesting birds. Ecology and Evolution, 6, 5907-5920.

Marteinson SC, Verreault J (2020) Changes in plasma biochemistry in breeding ring-billed gulls: Effects of anthropogenic habitat use and contaminant exposure. Environment International, 135, 105416.

Martin TE (1995) Avian life history evolution in relation to nest sites, nest predation, and food. Ecological Monographs, 65, 101-127.

Maziarz M, Broughton RK, Wesołowski T (2017) Microclimate in tree cavities and nest-boxes: Implications 
for hole-nesting birds. Forest Ecology and Management, 389, 306-313.

Melles S, Glenn SM, Martin K (2003) Urban bird diversity and landscape complexity: Species-environment associations along a multiscale habitat gradient. Conservation Ecology, 7, art5.

Meng XJ, Zhang KQ, Deng WH (2014) Nest site selection of black-naped oriole. Journal of Beijing Normal University (Natural Science), 50, 174-177. (in Chinese with English abstract) [孟晓静, 张克勤, 邓文洪 (2014) 黑枕黄鹂巢址 选择研究. 北京师范大学学报 (自然科学版), 50, 174-177.]

Mennechez G, Clergeau P (2006) Effect of urbanisation on habitat generalists: Starlings not so flexible? Acta Oecologica, 30, 182-191.

Mennerat A, Perret P, Lambrechts MM (2009) Local individual preferences for nest materials in a Passerine bird. PLoS ONE, 4, e5104.

Meyer LM, Schmidt KA, Robertson BA (2015) Evaluating exotic plants as evolutionary traps for nesting Veeries. The Condor, 117, 320-327.

Millones A, Frere E (2017) How nest site characteristics influence breeding success in red-legged cormorants Phalacrocorax gaimardi. Acta Ornithologica, 52, 239-244.

Mitrus C (2003) A comparison of the breeding ecology of collared flycatchers nesting in boxes and natural cavities. Journal of Field Ornithology, 74, 293-299.

Møller AP, Díaz M (2018) Avian preference for close proximity to human habitation and its ecological consequences. Current Zoology, 64, 623-630.

Moreno J, Martínez J, Corral C, Lobato E, Merino S, Morales J, la Puente JM, Tomás G (2008) Nest construction rate and stress in female pied flycatchers Ficedula hypoleuca. Acta Ornithologica, 43, 57-64.

Munro CA (1984) Roof nesting oystercatchers. Bird Study, 31, 148.

Mutzel A, Blom MPK, Spagopoulou F, Wright J, Dingemanse NJ, Kempenaers B (2013) Temporal trade-offs between nestling provisioning and defence against nest predators in blue tits. Animal Behaviour, 85, 1459-1469.

Narango DL, Tallamy DW, Marra PP (2017) Native plants improve breeding and foraging habitat for an insectivorous bird. Biological Conservation, 213, 42-50.

Olah G, Vigo G, Heinsohn R, Brightsmith DJ (2014) Nest site selection and efficacy of artificial nests for breeding success of Scarlet Macaws Ara macao Macao in lowland Peru. Journal for Nature Conservation, 22, 176-185.

Ortega YK, McKelvey KS, Six DL (2006) Invasion of an exotic forb impacts reproductive success and site fidelity of a migratory songbird. Oecologia, 149, 340-351.

Parece T, Campbell J (2018) Intra-urban microclimate effects on phenology. Urban Science, 2, 26.

Peach WJ, Vincent KE, Fowler JA, Grice PV (2008) Reproductive success of house sparrows along an urban gradient. Animal Conservation, 11, 493-503.

Pennington DN, Blair RB (2011) Habitat selection of breeding riparian birds in an urban environment: Untangling the relative importance of biophysical elements and spatial scale. Diversity and Distributions, 17, 506-518.

Provencher JF, Bond AL, Avery-Gomm S, Borrelle SB, Bravo Rebolledo EL, Hammer S, Kühn S, Lavers JL, Mallory ML, Trevail A, van Franeker JA (2017) Quantifying ingested debris in marine megafauna: A review and recommendations for standardization. Analytical Methods, 9, 1454-1469.

Qian SH, Qi M, Huang L, Zhao L, Lin DM, Yang YC (2016) Biotic homogenization of China's urban greening: A meta-analysis on woody species. Urban Forestry \& Urban Greening, 18, 25-33.

Radhamany D, Das KSA, Azeez PA, Wen LY, Sreekala LK (2016) Usage of nest materials by house sparrow (Passer domesticus) along an urban to rural gradient in Coimbatore, India. Tropical Life Sciences Research, 27, 127-134.

Rendell WB, Robertson RJ (1989) Nest-site characteristics, reproductive success and cavity availability for tree swallows breeding in natural cavities. The Condor, 91, 875-885.

Reynolds SJ, Davies CS, Elwell E, Tasker PJ, Williams A, Sadler JP, Hunt D (2016) Does the urban gradient influence the composition and ectoparasite load of nests of an urban bird species? Avian Biology Research, 9, 224-234.

Reynolds SJ, Ibáñez-Álamo JD, Sumasgutner P, Mainwaring MC (2019) Urbanisation and nest building in birds: A review of threats and opportunities. Journal of Ornithology, 160, 841-860.

Rodewald AD, Shustack DP, Hitchcock LE (2010) Exotic shrubs as ephemeral ecological traps for nesting birds. Biological Invasions, 12, 33-39.

Rodríguez J, Avilés JM, Parejo D (2011) The value of nestboxes in the conservation of Eurasian rollers Coracias garrulus in southern Spain. Ibis, 153, 735-745.

Rueegger N (2017) Artificial tree hollow creation for cavity-using wildlife-Trialling an alternative method to that of nest boxes. Forest Ecology and Management, 405, 404-412.

Scales J, Hyman J, Hughes M (2011) Behavioral syndromes break down in urban song sparrow populations. Ethology, 117, 887-895.

Schlaepfer MA, Runge MC, Sherman PW (2002) Ecological and evolutionary traps. Trends in Ecology \& Evolution, 17, 474-480.

Schlossberg S, King DI (2010) Effects of invasive woody plants on avian nest site selection and nesting success in shrublands. Animal Conservation, 13, 286-293.

Schnack S (1991) The breeding biology and nestling diet of the blackbird Turdus merula L. and the song thrush Turdus philomelos C. L. Brehm in Vienna and in an adjacent wood. Acta Ornithologica, 26, 85-106.

Schwartz T, Genouville A, Besnard A (2020) Increased 
microclimatic variation in artificial nests does not create ecological traps for a secondary cavity breeder, the European roller. Ecology and Evolution, 10, 13649-13663.

Seacor R, Ostovar K, Restani M (2014) Distribution and abundance of baling twine in the landscape near Osprey (Pandion haliaetus) nests: Implications for nestling entanglement. The Canadian Field Naturalist, 128, 173.

Seress G, Hammer T, Bókony V, Vincze E, Preiszner B, Pipoly I, Sinkovics C, Evans KL, Liker A (2018) Impact of urbanization on abundance and phenology of caterpillars and consequences for breeding in an insectivorous bird. Ecological Applications, 28, 1143-1156.

Shi LM, Liu NF, Ding W, Zhao QS, Fang FJ, Bao XK (2012) Nest-site selection and breeding success of the rufous-tailed shrike. Chinese Journal of Zoology, 47, 7-13. (in Chinese with English abstract) [施丽敏, 刘迺发, 丁未, 赵青山, 房 峰杰, 包新康 (2012) 荒漠伯劳巢址选择和繁殖成功. 动 物学杂志, 47, 7-13.]

Shochat E, Warren PS, Faeth SH, McIntyre NE, Hope D (2006) From patterns to emerging processes in mechanistic urban ecology. Trends in Ecology \& Evolution, 21, 186-191.

Shuttleworth CM (2001) Interactions between the red squirrel (Sciurus vulgaris), great tit (Parus major) and jackdaw (Corvus monedula) whilst using nest boxes. Journal of Zoology, 255, 269-272.

Sierro J, Schloesing E, Pavón I, Gil D (2017) European blackbirds exposed to aircraft noise advance their Chorus, modify their song and spend more time singing. Frontiers in Ecology and Evolution, 5, 68.

Sims V, Evans KL, Newson SE, Tratalos JA, Gaston KJ (2007) Avian assemblage structure and domestic cat densities in urban environments. Diversity and Distributions, 14, 387399.

Smith MD, Conway CJ, Ellis LA (2005) Burrowing owl nesting productivity: A comparison between artificial and natural burrows on and off golf courses. Wildlife Society Bulletin, 33, 454-462.

Soldatini C, Albores-Barajas YV, Mainardi D, Monaghan P (2008) Roof nesting by gulls for better or worse? Italian Journal of Zoology, 75, 295-303.

Sorace A (2002) High density of bird and pest species in urban habitats and the role of predator abundance. Ornis Fennica, 79, 60-71.

St Clair JJH, García-Peña GE, Woods RW, Székely T (2010) Presence of mammalian predators decreases tolerance to human disturbance in a breeding shorebird. Behavioral Ecology, 21, 1285-1292.

Stagoll K, Lindenmayer DB, Knight E, Fischer J, Manning AD (2012) Large trees are keystone structures in urban Parks. Conservation Letters, 5, 115-122.

Stinson LT, Pejchar L (2018) The effects of introduced plants on songbird reproductive success. Biological Invasions, 20, 1403-1416.

Suárez-Rodríguez M, Garcia CM (2017) An experimental demonstration that house finches add cigarette butts in response to ectoparasites. Journal of Avian Biology, 48, 1316-1321.

Suárez-Rodríguez M, Garcia CM (2014) There is no such a thing as a free cigarette; lining nests with discarded butts brings short-term benefits, but causes toxic damage. Journal of Evolutionary Biology, 27, 2719-2726.

Suárez-Rodríguez M, Montero-Montoya RD, Garcia CM (2017) Anthropogenic nest materials may increase breeding costs for urban birds. Frontiers in Ecology and Evolution, 5, 4.

Sun JJ, Wang SY, Wang YP, Shao DY, Ding P (2011) Effects of habitat fragmentation on avian nest predation risk in Thousand Island Lake, Zhejiang Province. Biodiversity Science, 19, 528-534. (in Chinese with English abstract) [ 孙 吉吉, 王思宇, 王彦平, 邵德钰, 丁平 (2011) 千岛湖栖 息地片段化效应对鸟类巢捕食风险的影响. 生物多样性, 19, 528-534.]

Sutherland DR, Dann P, Jessop RE (2014) Evaluation of artificial nest sites for long-term conservation of a burrownesting seabird. The Journal of Wildlife Management, 78, 1415-1424.

Teglhøj PG (2018) Artificial nests for barn swallows Hirundo rustica: A conservation option for a declining passerine? Bird Study, 65, 385-395.

Tian JD, Dong RJ, Lu JQ (2009) Nest-site selection of Pica pica in the new campus of Zhengzhou University. Journal of Henan Normal University (Natural Science), 37, 116-118. (in Chinese with English abstract) [田军东, 董瑞静, 路纪 琪 (2009) 郑州大学新校区喜鹊巢址选择研究. 河南师 范大学学报(自然科学版), 37, 116-118.]

Townsend AK, Barker CM (2014) Plastic and the nest entanglement of urban and agricultural crows. PLoS ONE, 9, e88006.

Tryjanowski P, Kosicki JZ, Kuźniak S, Sparks TH (2009) Long-term changes and breeding success in relation to nesting structures used by the white stork, Ciconia ciconia. Annales Zoologici Fennici, 46, 34-38.

Valera F, Václav R, Calero-Torralbo MÁ, Martínez T, Veiga J (2019) Natural cavity restoration as an alternative to nest box supplementation. Restoration Ecology, 27, 220-227.

Vigallon SM, Marzluff JM (2005) Abundance, nest sites, and nesting success of Steller's jays along a gradient of urbanization in Western Washington. Northwest Science, 79, 22-27.

Wang LX, Sui JL, Ma Q (2020) Influencing factors analysis of nest site selection and reproductive efficiency of Chinese sparrow hawk. Scientia Silvae Sinicae, 56, 116-122. (in Chinese with English abstract) [王龙祥, 隋金玲, 马强 (2020) 赤腹鹰巢址选择和繁殖成效的影响因子分析. 林 业科学, 56, 116-122.]

Wang YP, Chen SH, Blair RB, Jiang PP, Ding P (2009) Nest composition adjustments by Chinese bulbuls Pycnonotus sinensis in an urbanized landscape of Hangzhou (E China). 
Acta Ornithologica, 44, 185-192.

Wang YP, Chen SH, Ding P (2003) Breeding birds and their nests in street tree strips in Hangzhou City. Zoological Research, 24, 259-264. (in Chinese with English abstract) [王彦平, 陈水华, 丁平 (2003) 杭州城市行道树带的繁 殖鸟类及其鸟巢分布. 动物学研究, 24, 259-264.]

Wang YP, Chen SH, Jiang PP, Ding P (2008) Black-billed magpies (Pica pica) adjust nest characteristics to adapt to urbanization in Hangzhou, China. Canadian Journal of Zoology, 86, 676-684.

Wang YP, Huang Q, Lan SS, Zhang Q, Chen SH (2015) Common blackbirds Turdus merula use anthropogenic structures as nesting sites in an urbanized landscape. Current Zoology, 61, 435-443.

Wawrzyniak J, Kaliński A, Glądalski M, Bańbura M, Markowski M, Skwarska J, ZielińSki P, Cyżewska I, Bańbura J (2015) Long-term variation in laying date and clutch size of the great tit Parus majorin central Poland: A comparison between urban parkland and deciduous forest. Ardeola, 62, 311-322.

Wesołowski T (2011) Reports from nestbox studies: A review of inadequacies. Acta Ornithologica, 46, 13-17.

Wheeler MM, Neill C, Groffman PM, Avolio M, Bettez N, Cavender-Bares J, Roy Chowdhury R, Darling L, Grove JM, Hall SJ, Heffernan JB, Hobbie SE, Larson KL, Morse JL, Nelson KC, Ogden LA, O’Neil-Dunne J, Pataki DE, Polsky C, Steele M, Trammell TLE (2017) Continental-scale homogenization of residential lawn plant communities. Landscape and Urban Planning, 165, 54-63.

Wiebe KL (2011) Nest sites as limiting resources for cavity-nesting birds in mature forest ecosystems: A review of the evidence. Journal of Field Ornithology, 82, 239-248.

Wu JG (2004) Effects of changing scale on landscape pattern analysis: Scaling relations. Landscape Ecology, 19, 125-138.

Xie SL, Cao L, Lu F, Ouyang ZY (2016) Adaptation of birds to urbanization. Acta Ecologica Sinica, 36, 6696-6707. (in Chinese with English abstract) [谢世林, 曹垒, 逯非, 欧阳 志云 (2016) 鸟类对城市化的适应. 生态学报, 36, 6696-6707.]

Xu CZ, Guo ZR (2011) Research of adaptive nest-site selection by the red-tailed shrike (Lanius cristatus). Journal of Northeast Agricultural University, 42, 117-122. (in Chinese with English abstract) [徐纯柱, 郭自荣 (2011) 红尾伯劳 适应性巢址选择研究. 东北农业大学学报, 42, 117-122.]

Xu Y, Cao ZY, Wang B (2020) Effect of urbanization intensity on nest-site selection by Eurasian magpies (Pica pica). Urban Ecosystems, 23, 1099-1105.

Yan Y, Chai LY, Wu YJ, Dou L, Ran JH (2016) Breeding behavior of Garrulax sannio in Chengdu and its adaptation to the urban environments. Sichuan Journal of Zoology, 35, 936-941. (in Chinese with English abstract) [严勇, 柴璐艳, 吴永杰, 窦亮, 苒江洪 (2016) 成都市区白项噪鹛繁殖行 为及对城市环境的行为适应. 四川动物, 35, 936-941.]
Yang CC, Ye P, Huo J, Møller AP, Liang W, Feeney WE (2020) Sparrows use a medicinal herb to defend against parasites and increase offspring condition. Current Biology, 30, R1411-R1412.

Yang YW, Ci HX (2005) Studies on the adaptation of black-crowned night heron to urbanization in breeding season. Journal of Qufu Normal University (Natural Science), 31(3), 108-110. (in Chinese with English abstract) [杨月伟, 慈海金鍂 (2005) 繁殖期夜嘼对城市化的适应性 研究. 曲阜师范大学学报(自然科学版), 31(3), 108-110.]

Yu XW, Wang FY, Jiang Z, Huang SF, Hu XB (2014) Nest site selection of red-billed blue magpie (Urocissa erythrorhyncha). Chinese Journal of Wildlife, 35, 440-444. (in Chinese with English abstract) [于学伟, 王福云, 江志, 黄淑芳, 胡新波 (2014) 红嘴蓝鹊的巢址选择. 野生动物 学报, 35, 440-444.]

Zhang L, Zhang HW, Wang J, Zhang L, Cheng YT, Wan DM (2020) Nest site selection and breeding success of Daurian redstart Phoenicurus auroreus in northeast China. Acta Ecologica Sinica, 40, 70-76. (in Chinese with English abstract) [张雷, 张海旺, 王娟, 张丽, 程亚婷, 万冬梅 (2020) 东北地区北红尾鸲巢址选择及繁殖成效. 生态学 报, 40, 70-76.]

Zhang Q, Lan SS, Huang Q, Chen SH (2013) Urbanization effects on birds: From community to individual. Chinese Journal of Zoology, 48, 808-816. (in Chinese with English abstract) [张琴, 兰思思, 黄秦, 陈水华 (2013) 城市化对 鸟类的影响: 从群落到个体. 动物学杂志, 48, 808-816.]

Zhang SP, Zheng GM, Xu JL (2006) Habitat use of urban tree sparrows in the process of urbanization: Beijing as a case study. Biodiversity Science, 14, 372-381. (in Chinese with English abstract) [张淑萍, 郑光美, 徐基良 (2006) 城市 化对城市麻雀栖息地利用的影响: 以北京市为例. 生物 多样性, 14, 372-381.]

Zhao YL, Wang C, Bai ZT, Hao ZZ (2021) Changes of bird community under urbanization and its relationship with urban vegetation. Acta Ecologica Sinica, 41, 479-489. (in Chinese with English abstract) [赵伊琳, 王成, 白梓䑣, 郝 泽周 (2021) 城市化鸟类群落变化及其与城市植被的关 系. 生态学报, 41, 479-489.]

Zhi F, Yang TL, Zhang DB, Yan J (2018) Study of swallows nesting in urban areas of Wuhan City. Chinese Journal of Wildlife, 39, 334-339. (in Chinese with English abstract) [植飞, 杨天乐, 张东兵, 颜军 (2018) 武汉城区燕子繁殖 调查研究. 野生动物学报, 39, 334-339.]

Zhu X, Yang SD, Zou XP, Chen WZ, Ren JB (2000) The breeding habits and growth of the nestling black crowned night heron, Nycticorax nycticorax. Zoological Research, 21, 58-64. (in Chinese with English abstract) [朱曦, 杨士 德, 邹小平, 陈伟贞, 任金波 (2000) 夜鹭繁殖习性与生 长发育研究. 动物学研究, 21, 58-64.]

(责任编委: 王勇 责任编辑: 时意专) 